\title{
Detection of stain formation on teeth by oral antiseptic solution using fiber optic displacement sensor
}

\author{
H.A. Rahman, H.R.A.Rahim, S.W.Harun, M.Yasin, R.Apsari, H.Ahmad, W.A.BWanAbas
}

Introduction

In the last few decades, dental clinicians face increasing demands and expectations for accurate color matching due to the increasing attention given by the media and patients to esthetic dentistry. Hence, precise color matching has become an even more critical success factor for esthetic restoration. The subjective color perception of an observer leads to variations and unpredictable differences in color evaluation and matching among clinicians. Among the elements that affect the perceived color are the spectral distribution of a color stimulus, the surroundings of the stimulus [1], the state of an observer's visual system and on the observer's experience [2]. Furthermore, differ- ent surroundings lead to metamerism which is a phenomenon whereby different colors are exhibited by the same tooth when viewed under different lighting conditions [3].

Conventionally, clinicians used visual shade guides for tooth color matching. The use of shade guides is a quick and cost-effective method but its success depends strongly on professional experience [4]. Recently, significant advances have occurred in techniques and instruments for colorimetric analysis in dentistry which minimizes the subjective variance in the color matching process. These devices can be categorized as spectro photometers, colorimeters, digital color analyzers or combina tions of these. These devices have been demonstrated to be useful in quantifying color differences [5-9]. A spectrophotometer measures the spectral reflectance or transmittance curve of a specimen. In comparison to colorimeters, they have a longer working life but are more complex and expensive [5]. Colorimeters measure color tristimulus values from light reflected from the specimen. A series of photodiode filters are used to control light reaching the specimen. The repeatability of colorimeters may deteriorate with the aging of the filters [10]. Digital color analyzers have gained much popularity but the quality of the images are also influenced by lighting conditions and as such the integrity of the results are questionable [11].

Fiber optic displacement sensors (FODS) may potentially evolve into a new choice of dental color matching system featuring more advantages and potential. They are commonly constructed from multimode plastic optical fibers (POFs), which offer the benefit of low optical signal transmission loss, low production cost, compact size and compatibility with optical fiber technology. Three distinct methods are competent and commonly used for the displacement measurement: laser interferometry, wavelength modulation and the reflective intensity modulation technique. Laser interferometry [12] is based on fringe counting and offers high resolution and stability of measurement. However, its precision and stability are dependent on the wavelength of light. Wavelength modulation need fiber Bragg grating (FBG) and optical spectrum analyzer (OSA) for physical parameter detection and data acquisition, respectively, which are very costly. The intensity modulatedbased sensors use the modula tion of light power transmitted between the head of the sensor and the target surface [13]. In comparison with the first two methods for displacement measurements, the light intensity modulation is the simplest method to obtain a high resolution measurement. The intensity modulated sensors compete well with other sensing methods as they are relatively inexpensive, contactless, easy to be fabricated and suitable for employment in harsh environments. These type of sensors have been demonstrated to be efficient for measuring various types of surface profiles [14-16]. 
Most light intensity modulated displacement sensors operate by utilizing two parallel adjacent fibers (one for transmitting and the other for receiving light) [14-17] as they provide good sensing outcome. Polygerinos et al. [18] demonstrated the improved performance of an intensity modulated FODS by the use of a single optical fiber. Another research by Yasin et al. demonstrated the applicability of using concentric bundled fibers POFs with various number of receiving fibers and discovered that the sensitivity of a displacement sensor increases with the increasing number of receiving fibers within the bundled fiber [19]. This is mainly due to the increased total surface area on account of the increasing number of receiving fibers. He later attempted to compare the performances of a concentric bundled POF (consisting of one transmitting fiber and 16 receiving fibers) and a single transmitting and receiving fiber (multimode fused coupler) [20]. In terms of sensitivity, the former outperformed the latter by more than double its value but is inferior in terms of the operating displace- ment range. The use of concentric bundled POFs are especially advantageous in dental applications as they have rougher surface and tend to reflect light at various directions.

In the work presented here, we propose and demonstrate a simple intensity modulated fiber optic displacement sensor using a concentric bundled POF as well as teeth surfaces as reflecting targets. POFs are used as they offer the same advantages as conventional silica optical fibers but involve simpler and less expensive components. Furthermore, they possess greater flex-ibility and fracture toughness and the relatively large diameter $(1 \mathrm{~mm})$ of the multimode POF eases the handling and alignment process. In our approach, variations of colors are achieved via immersion of the teeth in different oral antiseptic solution concentration in de-ionized water as they are known for their negative effect in teeth staining [21-24]. This technique offers simplicity, reliability and continuous measurement capability. In addition, it is suitable for measuring color changes of teeth undergoing bleaching treatments.

Experimental setup

Fig. 1 shows the schematic diagram of the fiber optic displacement sensor which is employed for the detection of teeth stains. The sensor consists of a fiber optic transmitter, mechanical chopper, fiber optic probe, five flat human teeth surfaces, a silicon photo detector, lock-in amplifier and a computer. The fiber optic probe is made of two $2 \mathrm{~m}$ long polymethyl methacrylate (PMMA) which consists of one transmitting core of $1 \mathrm{~mm}$ in diameter and 16 receiving cores of $0.25 \mathrm{~mm}$ in diameter, numerical aperture of 0.5 , core refractive index of 1.492 and cladding refractive index of 1.402. The output signal (reflected signal) is the power sum of each of the receiving fibers [25]. A red He-Ne laser(l1/4633nm) was used as the light source with an average output power of $5.5 \mathrm{~mW}$, beam diameter of $0.80 \mathrm{~mm}$ and beam divergence of $1.01 \mathrm{mrads}$. The photodetector is a high speed silicon photodiode with an optical response of 400-1100 nm, making it compatible with a wide range of visible light including the $633 \mathrm{~nm}$ visible red He-Ne laser used in this setup. The light source was modulated externally by a chopper with a frequency of $113 \mathrm{~Hz}$ as to avoid the harmonics from the line frequency which is about $50-60 \mathrm{~Hz}$. The modulated light source was used in conjunction with a lock-in amplifier to reduce the dc drift and interference of ambient stray light.

The displacement of the fiber optic probe was achieved by mounting it on a micrometer translation stage, which was rigidly attached to a vibration free table. Light from the fiber optic transmitter (peak wavelength at $633 \mathrm{~nm}$ ) was coupled into the transmitting core. The signal from the receiving cores was measured by moving the probe away from the zero point, where the reflective surface of the teeth samples and the probe were in close contact. The signal from the detector was converted to voltage and measured by a lock-in amplifier and computer via RS232 using a Delphi software.

Five human teeth, namely canine teeth were extracted and stored in water at room temperature one week prior to the experiments. The teeth surfaces were cut to produce smooth and flattened surfaces of equal height. The probe can be adjusted to obtain the maximum reflected light as to ensure that the probe and the tooth surface are perpendicular to each other. One of the teeth samples was used as the control sample while the other four were soaked for a duration of $24 \mathrm{~h}$ in oral antiseptic solutions (Bactidol, $0.1 \%$ hexetidine, Interphil Laboratories Inc., Singapore) with concentrations of 20, 40, 60 and $80 \mathrm{ml}$ diluted with de- ionized water to a total volume of $100 \mathrm{ml}$. Each of the five teeth samples was used consecutively as the reflecting target whilst measuring the output intensity by changing the position of the fiber optic probe from 0 to $2.5 \mathrm{~mm}$ in a step of $50 \mathrm{~mm}$. There was no actual contact between the probe and the tooth surface as the zero 
displacement actually refers to a very small distance between them. In preparation for the experiment, the teeth were removed from the antiseptic solution and left dried without being washed. During the experiment, the temperature was kept constant at $25 \mathrm{C}$ and the error due to this temperature variation is negligible. The use of the same type of teeth surfaces and teeth dimension reduces the error due to variation in teeth surface roughness.

Full text is available at :

http://ac.els-cdn.com/S0030399212002952/1-s2.0-S0030399212002952-main.pdf? tid=c3fdf6e6-a017-11e3a2a5-00000aacb35d\&acdnat $=1393551148 \_8 e d c f f 84 b 1$ b3fda07effea7f062800a5

http://www.sciencedirect.com/science/article/pii/S0030399212002952 